\title{
Monitoring observations of the interaction between Sgr A* and G2 with the Karl G. Jansky Very Large Array
}

\author{
Loránt O. Sjouwerman and Claire J. Chandler \\ National Radio Astronomy Observatory, P. O. Box O, Socorro, NM 87801, USA \\ email: lsjouwer@nrao.edu, cchandle@nrao.edu
}

\begin{abstract}
We report on an ongoing community service observing program to follow the expected encounter of the G2 cloud with the black hole Sgr A* in 2013. The NRAO Karl G. Jansky Very Large Array (VLA) has been observing the Sgr A region since 2012 October on roughly a bi-monthly interval, each for two hours, cycling through eight observing bands at their default continuum frequencies, using $2 \mathrm{GHz}$ of bandwidth. The data from the monitoring program are publicly available through the NRAO data archive immediately after observing has completed, and the flux densities are published by NRAO staff as soon as the data are reduced. The cumulative results of the monitoring effort are posted on the service observing web page https://science.nrao.edu/science/service-observing and so far do not indicate a significant brightening of the emission from the direction of Sgr A* over the period 2012 October to 2013 September, within the calibration uncertainties.
\end{abstract}

\section{Motivation}

In 2012, after an ad hoc committee of community members was asked by the director what NRAO could provide to observe the upcoming impact of the G2 gas cloud with Sgr A*, NRAO responded to the committee's "Recommendations for the Optimal NRAO Response to the G2 Encounter with Sgr A*" report with the following actions and results. The scientific goal is to provide measurements with both the VLA and VLBA to characterize the emission before the encounter, and monitoring with the VLA during the encounter, extending through April 2014. All data and data products are non-proprietary and made available to the community in a timely fashion, which then can be used to trigger follow-up observations.

VLA monitoring observations cover $2 \mathrm{GHz}$ bandwidth at all standard continuum bands $(1.5,3.0,5.5,9.0,14.0,21.2,32$, and $41 \mathrm{GHz})$ in 2-hour scheduling blocks without polarization calibration. The initial cadence started with observing every two months from 2012 October (A array configuration), with monthly observations closer to the transit. To date (2013.09.29) nine of a planned 13 epochs have been successfully observed under program TOBS0006 and are publicly available in the NRAO data archive (Figure 1).

\section{Current trends}

Figure 2 on the bottom displays the trending of the flux density of Sgr A* during the monitoring to date. The data are normalized by the mean flux density of all nine epochs, per frequency band, to capture the relative change in flux over time in a single plot. There is significant extended emission from the Galactic center region, and only upper limits can be obtained for the lower frequencies in the compact array configurations, so this analysis has only been carried out for the higher frequencies. A flux density increase over 

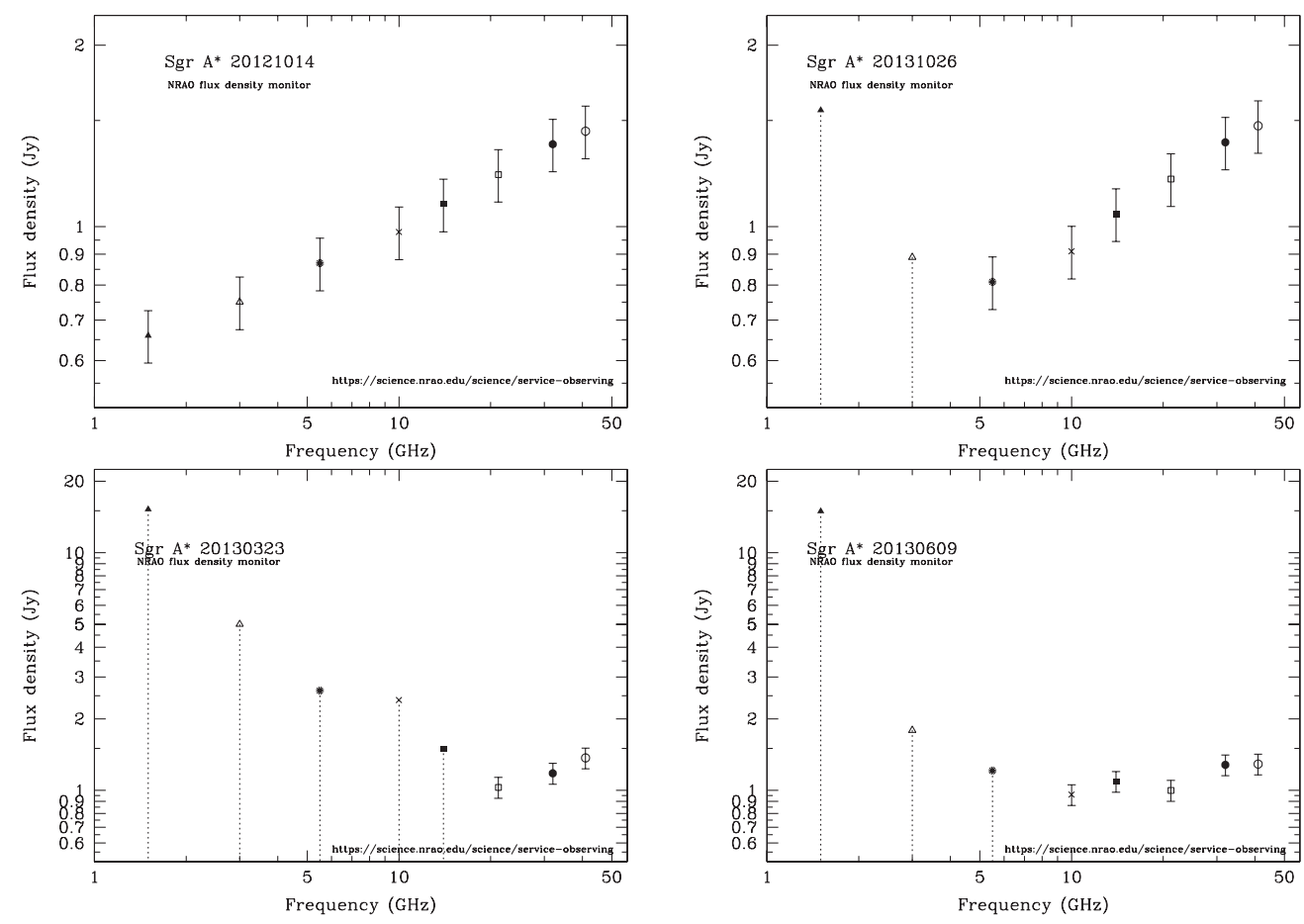

Figure 1. Flux density measurements of Sgr A*: Top left: First 2012 October (A array configuration), Top right: Latest 2013 October (B array configuration). Monitoring data taken in the more compact array configurations (like in D and C array configuration, bottom left and right) with lower angular resolution, suffer severely from confusion at the lower frequencies. Only upper limits are available at these bands.

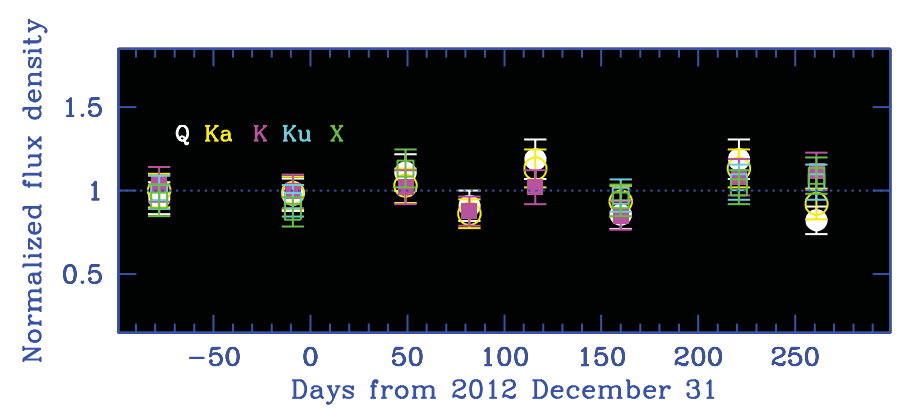

Figure 2. Trending of the normalized flux density of $\mathrm{Sgr} \mathrm{A}^{*}$ during the monitoring to date. Small deviations from unity are within the measurement errors or can be explained by systematic effects such as weather; there are no systematic temporal flux density trends. [A COLOR VERSION IS AVAILABLE ONLINE.]

time would show as a flux density significantly lower than the average (unity) at earlier times and a flux significantly higher than the average at later times. A small relative error in the absolute flux density measurements (e.g., due to weather or elevation effects) would show all flux density measurements from a single epoch to shift similarly from the average. The small deviations observed here are probably due to such measurement errors. All measurements to date are consistent with the mean to within $2 \sigma$. 
The VLA monitoring shows no significant change in the flux density measured between 10 and $45 \mathrm{GHz}$ in the period from 2012 October to 2013 October.

These VLA monitoring data products can be obtained from the NRAO service observing web page at https://science.nrao.edu/science/service-observing. 